\title{
Oxidative Stress and Changes on the Adenosinergic System of Cats Infected by Feline Leukemia Virus (FeLV)
}

\author{
Giovana Biezus ', Renata Assis Casagrande', Matheus Dellaméa Baldissera' ${ }^{2}$, Nathieli Bianchi Bottari ${ }^{3}$, \\ Paulo Eduardo Ferian' ${ }^{1}$, Vera Maria Morsch ${ }^{3}$, Maria Rosa Chitolina Schetinger ${ }^{3}$, Jéssica Aline Withoeft ${ }^{1}$, \\ Leonardo Henrique Hasckel da Silva Pereira' ${ }^{1}$, Aleksandro Schafer da Silva ${ }^{3,4}$ \& Gustavo Machado ${ }^{5}$
}

\begin{abstract}
Background: The feline leukemia virus (FeLV) is clinically important retroviruses that infect domestic and wild feline worldwide, affecting more than 3 million cats representing a great risk for premature death. Some studies have demonstrated that oxidative stress, as well as the adenosinergic system, exert an important role in the pathogenesis of viral diseases. Oxidative stress is considered a disturbance in the antioxidant/oxidant status in favor of the excessive generation or lower removal of free radicals. Therefore, the aim of this study was to evaluate whether changes on the adenosinergic system and oxidative stress occurred in cats positives for feline leukemia virus (FeLV).

Materials, Methods \& Results: Forty-nine serum samples of cats (between 4 months and 13 years of age) seen at the Veterinary Hospital of the University of Santa Catarina State (UDESC - Lages, SC, Brazil) were used. Blood samples were collected from the jugular vein and stored in tubes without anticoagulant to obtain serum. An aliquot was used to detect both viral infections using the kit SNAP FIV/FeLV, that detects the p27 protein from FeLV and antibodies against the p24 protein from FIV. The test was performed according the manufacture's recommendations. Based on this result, this study involved $20 \mathrm{FeLV}$ negative cats, $20 \mathrm{FeLV}$ positive symptomatic cats, and $9 \mathrm{FeLV}$ positives asymptomatic cats. Serum samples were used to determine the activities of adenosine deaminase (ADA) and glutathione-S-transferase (GST), as well as thiobarbituric acid reactive substances (TBARS) and reactive oxygen levels (ROS). Seric ADA and GST activities, as well as TBARS (lipid peroxidation) and ROS (free radical) levels were determined spectrophotometrically according to the specific method. Seric ADA activity was increased in symptomatic animals compared to negatives and also to asymptomatic positive animals, and the same was observed for seric TBARS levels. On the other hand, GST activity decreased in the symptomatic and asymptomatic groups compared to the negative control group. No difference was observed regarding seric ROS levels.

Discussion: The upregulation of seric ADA activity observed in this present study could contribute to the inflammatory process since it would decrease seric levels of Ado, a nucleoside with anti-inflammatory effects. Lipids, especially the polyunsaturated fatty acids, are sensitive to oxidation by free radicals, generating MDA, and cause to increase of TBARS levels. A significant negative correlation between lipid peroxidation and ADA activity in kidney samples of rats experimentally infected by protozoan, and these authors demonstrated that increase on lipid peroxidation is linked to decreased ADA activity. The reduction on seric GST activity can be explained since the antioxidant enzyme was unable to remove the excess of peroxides and superoxide anion, resulting on decreased cellular antioxidant activity. Based on these evidences, it is possible to conclude that symptomatic FeLV cats showed an upregulation on seric ADA activity associated to the impairment of the immune response, contributing to inflammatory processes. Moreover, FeLV symptomatic cats showed damage to lipids and an impairment on antioxidant/oxidant status, alterations compatible to oxidative stress and oxidative damage. In summary, these alterations contribute to the pathophysiology of FeLV infection, and can be used as markers of this disease.
\end{abstract}

Keywords: FeLV, oxidants, antioxidants, ADA.

Received: 5 May 2017

Accepted: 3 October 2017

Published: 24 October 2017

${ }^{1}$ Department of Veterinary Medicine, Universidade do Estado de Santa Catarina (UDESC), Lages, SC, Brazil. ${ }^{2}$ Department of Microbiology and Parasitology \& ${ }^{3}$ Graduate Program in Toxicological Biochemistry and Department of Biochemistry and Molecular Biology, Universidade Federal de Santa Maria (UFSM), Santa Maria, RS, Brazil. ${ }^{4}$ Department of Animal Science, UDESC, Chapecó, SC. ${ }^{5}$ Veterinary Population Medicine Department (VPM), University of Minnesota, St. Paul, MN, USA. CORRESPONDENCE: R.A. Casagrande [renata.casagrande@udesc.br - Tel +55 (49) 32899160]. Departamento de Medicina Veterinária, Universidade do Estado de Santa Catarina (UDESC). Av. Luiz de Camões n. 2090. Bairro Conta Dinheiro. CEP 88520-000 Lages, SC, Brazil. 


\section{INTRODUCTION}

The feline immunodeficiency virus (FIV) and the feline leukemia virus (FeLV) are clinically important retroviruses that infect domestic and wild feline worldwide, affecting more than 3 million cats representing a great risk for premature death $[3,13]$. FeLV infection may present different outcomes, such as progressive form, commonly acquired via the oronasal route by mutual grooming, nursing, bites or food sharing, and adult males with outdoor access are at higher risk for acquiring this infection [13]. Progressive infection can induce the development of neoplasms such as lymphoma, hematopoietic and neurological disorders, immunodeficiencies, and stomatitis [7], that contribute to premature death. Recently, some studies have demonstrated that oxidative stress, as well as the adenosinergic system, exert an important role in the pathogenesis of viral diseases, such as observed by researchers in mice infected by herpes simplex virus 2 (HSV-2) [17].

Oxidative stress is considered a disturbance in the antioxidant/oxidant status in favor of the excessive generation or lower removal of free radicals [20], that can lead to an oxidation of biomolecules with consequent loss of their biological function, contributing to disease pathogenesis of viral infections [17]. The involvement of oxidative stress during FeLV infection remains poorly understood, limited only to an oxidative effect on peripheric leukocytes of naturally infected cats [9]. Similarly, the adenosinergic system, through the enzyme adenosine deaminase (ADA) is considered a novel marker for inflammatory process during infectious diseases [18]. ADA, as a key enzyme in the purine metabolism, catalyzes the irreversible deamination of adenosine (Ado) into inosine, regulating the extracellular Ado levels, a molecule with antiinflammatory profile [4]. Based on these evidences, the aim of this study was to evaluate whether changes on the adenosinergic system and oxidative stress occurred in FeLV positive cats.

\section{MATERIALS AND METHODS}

\section{Animals}

In this study, 49 serum samples of cats (between 4 months and 13 years of age) seen at the Veterinary Hospital of the University of Santa Catarina State (UDESC - Lages, SC, Brazil) were used. Blood samples were collected from the jugular vein, and stored in tubes without anticoagulant to obtain serum, divided into two micro tubes. An aliquot of serum was used for diagnostic serological tests. The second aliquot of sera was used to perform the biochemical analyses.

\section{Serological examinations and experimental group}

In the laboratory, the tubes were centrifuged at $2000 \mathrm{x} g$ during $10 \mathrm{~min}$. The serum was divided into two microtubes and stored at $-80^{\circ} \mathrm{C}$. An aliquot was used to detect both viral infections using the kit SNAP FIV/FeLV (Combo test $\left.{ }^{\circledR}\right)^{1}$, that detects the p27 protein from FeLV and antibodies against the p24 protein from FIV. The test was performed according the manufacture's recommendations.

The second aliquot of sera was used to perform the biochemical analyses.

\section{Seric ADA analysis}

Seric ADA activity was determined spectrophotometrically according to the method described in details [2]. The specific activity was reported as U/L.

\section{Seric lipid peroxidation analysis}

Seric lipid peroxidation was determined by TBARS levels [11]. The reaction product was measured spectrophotometrically at $532 \mathrm{~nm}$, and the results were expressed as nanomoles of malondialdehyde by milliliter of serum (nmol/MDA $/ \mathrm{mL}$ of serum). Seric reactive oxygen species (ROS) levels were determined as an index from peroxide produced in the cellular components via DCFH production on the oxidation assay [12]. The absorbance was measured by a spectrophotometer at $522 \mathrm{~nm}$. The results were expressed as $\mathrm{U} \mathrm{DCF} / \mathrm{mL}$ of serum.

\section{Seric GST analysis}

Seric glutathione-S-transferase (GST) activity was measured using the assay based on the biochemical method [5]. The absorbance was measured at timed intervals, and the specific GST activity of each lysate was determined based on the increase of absorbance, the time between measurements ( $\mathrm{min}$ ), and the amount of protein $(\mu \mathrm{g})$. Seric GST activity as calculated as $\mu \mathrm{mol}$ of $\mathrm{Cdnb} / \mathrm{min} / \mathrm{mg}$ of protein.

\section{Statistical analysis}

For each group all parameters were tested for normality using the Shapiro-Wilk test, and the skew- 
ness, kurtosis and homogeneity by the Levene test, and log transformation was applied when needed. A one-way ANOVA was used to analyze all parameters, and post hoc test Tukey test was usedaccordingly. Significantly different was assumed if $P<0.05$. The statistical process was performed using R-language, v.3.1 (R Development Core Team 2012).

\section{RESULTS}

Among the selected animals, 20 cats were negatives for both diseases (used in the control group);
20 cats were positives for FeLV (symptomatic animals with anemia, immunosuppression and lymphoma), and 9 cats were positives for FeLV (asymptomatic animals).

Seric ADA activity was increased in symptomatic animals compared to negatives and also to asymptomatic positive animals (Figure 1), and the same was observed for seric TBARS levels (Figure 2A). On the other hand, GST activity decreased in the symptomatic and asymptomatic groups compared to the negative control group (Figure 2B). No difference was observed regarding seric ROS levels (Figure 2C).

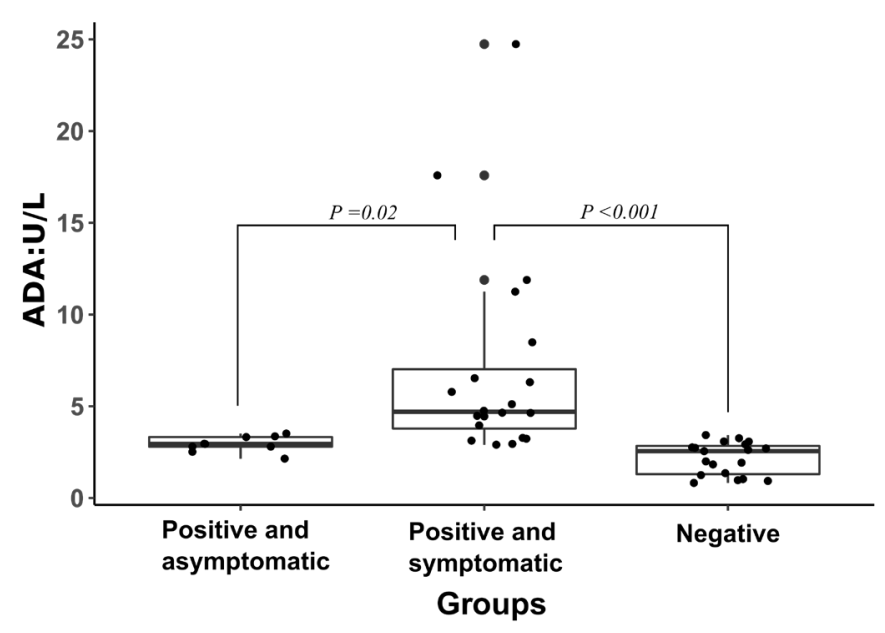

Figure 1. Seric adenosine deaminase (ADA) activity in symptomatic and asymptomatic positive cats afterfeline leukemia virus (FeLV) infection compared to negative cats using the Tukey post hoc test.
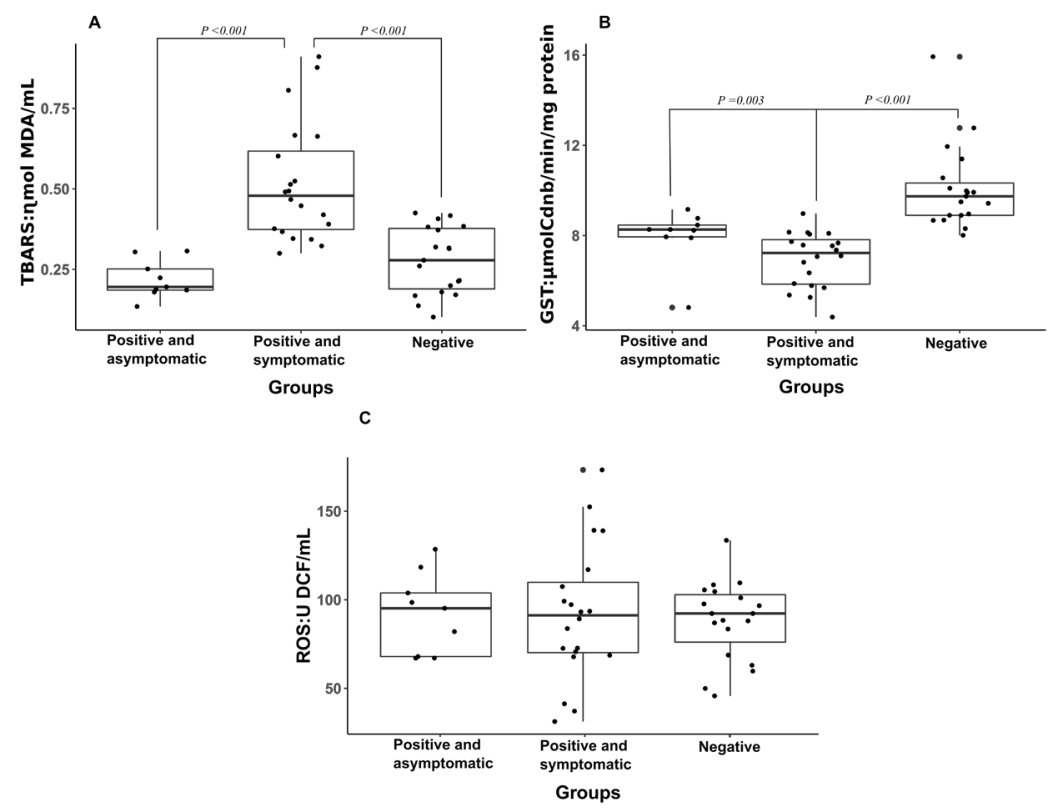

Figure 2. Seric levels of thiobarbituric acid reactive substances (TBARS) [A], activity of glutathione S-transferase (GST) [B] and reactive oxygen levels (ROS) [C] in symptomatic and asymptomatic positive cats afterinfection by feline leukemia virus (FeLV) compared to negative cats using the Tukey post hoc test. 


\section{DISCUSSION}

The upregulation of seric ADA activity observed in this present study could contribute to the inflammatory process since it would decrease seric levels of Ado, a nucleoside with anti-inflammatory effects, in disagree to what was observed in other report [1]. A study demonstrated a significant reduction on seric total ADA activity, as well in the seric ADA1 and ADA2 activities in cats positives for FeLV [1]. According to these authors, the downregulation of seric ADA activity works as an attempt to restore the immunity by increasing Ado levels, and this response is linkedto the immunosuppressive effect of the protein $\mathrm{p} 15 \mathrm{E}$ during chronic infections by retrovirus [10] and to functional defects of peripheral T-lymphocytes [16]. In our study, the upregulation on ADA activity might be correlated to injured tissues and cells, i.e, with oxidative stress [2,19]. In summary, the upregulation on seric ADA activity exerts a pro-inflammatory profile that contributes to the impairment of the immune response, and it may be linked to oxidative stress.

A significant increase in TBARS levels was observed in serum of symptomatic cats for FeLV. Lipids, especially the polyunsaturated fatty acids, are sensitive to oxidation by free radicals, generating MDA [6]. To the best of our knowledge, there are no reports regarding the seric lipid peroxidation in symptomatic and/ or asymptomatic cats for FeLV, but there are several evidences of lipid peroxidation caused by retroviruses [14]. Recently, a study demonstrated a significant negative correlation between lipid peroxidation and ADA activity in kidney samples of rats experimentally infected by Trypanosoma evansi [2], and these authors demonstrated that increase on lipid peroxidation is linked to decreased ADA activity, similarly to what was observed in this present study. In summary, lipid peroxidation contributes to disease pathogenesis of FeLV through oxidative and inflammatory pathways. The lipid peroxidation significantly increased with concomitant depletion of GST activity, that acts for metabolism detoxification [8]. This reduction on seric GST activity can be explained since the antioxidant enzyme was unable to remove the excess of peroxides and superoxide anion, resulting on decreased cellular antioxidant activity [15].

\section{CONCLUSIONS}

Based on these evidences, symptomatic cats infected by FeLV showed an upregulation on seric ADA activity that is associated to the impairment of immune responses, contributing to inflammatory processes. Moreover, symptomatic cats infected by FeLV showed damage to lipids and an impairment on antioxidant/oxidant status, alterations compatible to oxidative stress and oxidative damage. In summary, these alterationson ADA activity and oxidative stress contribute to the pathophysiology of FeLV.

\section{MANUFACTURER}

${ }^{1}$ Idexx Laboratories. Westbrook, ME, USA.

Ethical approval. The experiment was approved by the Ethics Committee on Animal Research of the Universidade do Estado de Santa Catarina (UDESC), under protocol number 7932191015.

Declaration of interest. The authors report no conflicts of interest. The authors alone are responsible for the content and writing of the paper.

Acknowledgments. This work was financially supported by the Brazilian National Council of Scientific and Technologic Development (CNPq, Universal 407084/2016-9) and by the FAPESC (01/2016, 2017TR647).

\section{REFERENCES}

1 Altug N., Yuksek N., Agaoglu Z.T. \& Ozkan C. 2007. Decreased serum adenosine deaminase activities in van cats with feline retroviruses infections. Medycyna Weterynaryjna. 63(2): 184-186.

2 Baldissera M.D., Souza C.F., Grando T.H., Da Silva A.S. \& Monteiro S.G. 2016. Involvement of oxidative stress, cholinergic and adenosinergic systems on renal damage caused by Trypanosoma evansi infection: Relationship with lipid peroxidation. Microbial Pathogenesis. 99(2): 191-195.

3 Filoni C., Catão-Dias J.L., Cattori V., Willi B., Meli M.L., Corrêa S.H., Marques M.C., Adania C.H., Silva J.C., Marvulo M.F., Ferreira Neto J.S., Durigon E.L., de Carvalho V.M., Coutinho S.D., Lutz H. \& Hofmann-Lehmann R. 2012. Surveillance using serological and molecular methods for the detection of infectious diseases agents in captive Brazilian neotropic and exotic felids. The Journal of Veterinary Diagnostic Investigation. 24(2): 166-173.

4 Franco R., Casado V., Ciruela F., Saura C., Mallo J., Canela E.I. \& Louis C.1997. Cell surface adenosine deaminase: much more than an ectoenzyme. Progression Neurobiology. 52(3): 283-294. 
5 Habig W.H., Pabst M.J. \& Jakoby W.B. 1974. Glutathione S-transferase. The first enzymatic step in mercapturic acid formation. Journal of Biological Chemistry. 249(22): 7130-7139.

6 Halliwell B. \& Gutteridge J.M.C. 2007. Free Radicals in Biology and Treatment. Oxford: Oxford University Press, 100 p.

7 Hartmann K. 2012. Clinical aspects of feline retroviruses: A Review. Viruses. 4(11): 2684-2710.

8 Hayes J., Flanagan J. \& Jowsey I. 2005. Glutathione transferases. Annual Review of Pharmacology and Toxicology. 45(1): 51-88.

9 Hoffmann-Jagielska M., Winnicka A., Jagielski D., Micun J., Zmudzka M. \& Lechowski R. 2005. Influence of naturally acquired feline leukemia virus (FeLV) infection on the phagocytic and respiratory burst activity of neutrophils and monocytes of peripheral blood. Polish Journal of Veterinary Sciences. 8(1): 93-97.

10 Hofmann-Lehmann R., Holznagel E., Ossent P. \& Lutz H. 1997. Parameters of disease in long-term experimental feline retrovirus (feline immunodeficiency virus and feline leukemia virus) infections: hematology, clinical chemistry and lymphocyte subsets. Clinical and Diagnostic Laboratory Immunology. 4(1): 33-42.

11 Jentzsch A.M., Bachmann H., Furst P. \& Biesalski H.K. 1996. Improved analysis of malondialdehyde in human body fluids. Free Radical Biology and Medicine. 20(2): 251-256.

12 Lawler J.M., Song W. \& Demaree S.R. 2003. Hind limb unloading increases oxidative stress and disrupts antioxidant capacity in skeletal muscle. Free Radical Biology and Medicine. 35(1): 9-16

13 Levy J., Crawford C., Hartmann K., Hofmann-Lehmann R., Litle S. \& Sundahl E. 2008. American association of feline practitioner's feline retrovirus management guidelines. Journal of Feline Medicine and Surgery. 10(3): 300-316.

14 Liang B., Zhang Z., Araghiniknam M., Eskelson C. \& Watson R.R. 1997. Prevention of retroviruses-induced aberrant cytokine secretion, excessive lipid peroxidation, and tissue vitamin E deficiency by T cell receptor peptide treatments in C57BL/6 mice. Proceedings of the Society for Experimental Biology and Medicine. 214(1): 87-94.

15 Lushchak V.I. 2011. Environmentally induced oxidative stress in aquatic animals. Aquatic Toxicology. 101(2011): $13 \mathrm{e} 30$.

16 Renouf J.A., Wood A., Frazer I.H., Thong Y.H. \& Chalmers A.H. 1989. Depressed activities of purine enzymes in lymphocytes of patients infected with human immunodeficiency virus. Clinical Chemistry. 35(11): 1478-1481.

17 Sartori G., Jardim N.S., Sari M.H., Flores E.F., Prigol M. \& Nogueira C.W. 2017. Diphenyl diselenide reduces oxidative stress and toxicity caused by HSV- infection in mice. Journal of Cellular Biochemistry. 118(9): 1028-1037.

18 Tonin A.A., Calado A.M.C., Bottari N.B., Dalenogare D., Thomé G.R., Duarte T., Duarte M.M.M.F., Morsch V.M., Schetinger M.R.C., Alves L.C., Tinucci-Costa M. \& Da Silva A.S. 2016. Novel markers of inflammatory response and hepatic dysfunction in canine leishmaniasis. Comparative Immunology, Microbiology and Infectious Diseases. 44(1): 61-64.

19 Ungerer J.P.J., Oosthuizen H.M., Retief J.H. \& Bissbort S.H. 1994. Significance of adenosine deaminase activity and its isoenzymes in tuberculous effusions. Chest. 106(1): 33-37.

20 Winterbourne C.C. 2015. Are free radicals involved in thiol-based redox signaling? Free Radical Biology and Medicine. 80(1): 164-170. 